\title{
Biología reproductiva de la tagua común (Fulica armillata) y la tagua de frente roja ( $F$. rufifrons) en un área agroforestal del centro-sur de Chile
}

\section{Breeding biology of red-gartered coot (Fulica armillata) and red-fronted coot (F. rufifrons) in an agroforestry area of central-south Chile}

\author{
Carolina Silva ${ }^{1}$, Carlos Barrientos ${ }^{1}$, Ricardo A. Figueroa R. ${ }^{2}$, Nicolás Martín ${ }^{1}$, Álex Contreras ${ }^{1}$, \\ Karen Ardiles ${ }^{1}$, Lucila Moreno ${ }^{3}$ \& Daniel GonzÁlez-Acuña ${ }^{1}$
}

${ }^{1}$ Facultad de Ciencias Veterinarias, Universidad de Concepción, Departamento de Ciencias Pecuarias, Casilla 537, Chillán, Chile.

E-mail: danigonz@udec.cl

${ }^{2}$ Escuela de Postgrado, Facultad de Ciencias, Universidad de Austral, Valdivia, Chile.

${ }^{3}$ Facultad de Ciencias Naturales y Oceanográficas, Universidad de Concepción, Departamento de Zoología, Casilla 160-C, Concepción, Chile.

\begin{abstract}
RESUMEN
Entre los periodos reproductivos 2008 y 2009, se estudió la nidificación y reproducción de Fulica armillata y F. rufifrons que habitan una laguna natural en un área agroforestal del centro-sur de Chile. Se estudiaron las diferencias en las características de los nidos, tamaño de las puestas, características de los huevos, periodo de incubación, éxito de eclosión y características de los pichones, con el objetivo de aportar información básica sobre la ecología reproductiva de estas especies. En ambas especies el periodo reproductivo se inició a fines de septiembre, extendiéndose hasta finales de enero. Las plataformas de nidificación de ambas taguas fueron de tamaño similar y estuvieron compuestas de juncos secos entrecruzados siguiendo un patrón circular, con una taza poco profunda. Los tamaños de nidada de F. rufifrons casi doblaron a los de $F$. armillata. Los huevos de $F$. armillata se distinguieron de los huevos de $F$. rufifrons por su tamaño, peso y patrón de coloración. El período de incubación en F. armillata y F. rufifrons fue 27 y 26 días, respectivamente. Las características de los pichones variaron entre especies, presentando diferencias evidentes en sus patrones de coloración. En general, nuestras observaciones coinciden con antecedentes previos acerca de la biología reproductiva de las especies de taguas estudiadas. La descripción de huevos y pichones hechas aquí puede ser una guía útil para diferenciar los nidos de F. armillata y F. rufifrons. Además, parte de nuestros resultados puede tener utilidad potencial para planificar el manejo y conservación de ecosistemas dulceacuícolas.
\end{abstract}

Palabras clave: éxito de eclosión, Chile, nidos, tagua, reproducción.

\begin{abstract}
Between the breeding seasons 2008 and 2009 we studied the nesting and breeding of Fulica armillata and F. rufifrons inhabiting a natural lagoon in an agro-forestry area of central-south Chile. We studied the differences in characteristics of nest, eggs, incubation, hatching success and characteristics of the young, with the aim provide basic data on breeding ecology of coots in Chile. In both species the breeding period started in late September, extending into late January. Nesting platforms of both coots were similar in size and built with dried crossed juncus, had a circular form and shallow nest-cup. Clutch sizes of $F$. rufifrons were almost two-fold those of $F$. armillata. The eggs of $F$. armillata distinguished from the eggs of F. rufifrons by size, weight and color pattern. Incubation time in F. armillata and F. rufifrons was 27 and 26 days, respectively. $F$. armillata had a hatching success of $46 \%(88.5 \%$, traditional method) and $62 \%$ (96\%) for 2008 and 2009 , respectively; while for F. rufifrons was $85 \%$ (98\%) for 2008. Although chicks of both species born with black downs, they exhibited an evidently different coloration pattern. In general, our observations agree with previous reports on the breeding biology of the studied coots. We believe that the description of eggs and chicks made here may become a useful guide to recognize nests of F. armillata and F. rufifrons. In addition, some of our results may have potential utility for management and conservation planning of freshwater ecosystems.
\end{abstract}

KEY wORDS: breeding, Chile, coot, hatching success, nest. 


\section{INTRODUCCIÓN}

El estudio de la biología reproductiva de las aves dulceacuícolas es esencial para generar información que permita evaluar las adaptaciones y tolerancia de estos organismos al impacto humano $\mathrm{y}$, a la vez, mitigar los efectos negativos que pueden generar la transformación de los ecosistemas naturales (Kushlan 1993; Weller 1999).

La tagua común (Fulica armillata Vieillot 1817) y la tagua de frente roja (Fulica rufifrons Philippi \& Landbeck 1861) son especies características de humedales dulceacuícolas cuya distribución comprende el sudeste de Brasil, Uruguay, Paraguay, Argentina y Chile. En Chile, F. armillata se distribuye ampliamente desde Coquimbo hasta Tierra del Fuego e Isla Navarino (30-56 ${ }^{\circ} \mathrm{S}$; Wetmore 1926; Housse 1945; Couve \& Vidal 2000; Figueroa et al. 2001). En cambio, la distribución de $F$. rufifrons parece ser más restringida. Aunque ésta ha sido registrada accidentalmente en Tierra del Fuego e Isla Robinson Crusoe, su rango estable de distribución abarca desde Copiapó hasta el Seno de Reloncaví (27-42 ${ }^{\circ}$; Jaramillo et al. 2003; Martínez \& González 2005). Si bien en Chile ambas especies son ubicuas en gran parte de su rango de distribución (Couve \& Vidal 2003; Jaramillo et al. 2003), ellas presentan diferencias conductuales. Mientras la tagua común forma grupos conspicuos y numerosos, la tagua de frente roja tiende a ocultarse entre la vegetación y a entremezclarse con los grupos de otras especies de taguas (Rottmann 1995; Couve \& Vidal 2003; Jaramillo et al. 2003).

A pesar de ser especies comunes, la biología reproductiva de F. armillata y F. rufifrons es conocida sólo por descripciones breves de sus nidos, huevos, tamaño de nidada, período de postura (Housse 1945; Goodall et al. 1951; Fjeldså \& Krabbe 1990; Rottmann 1995; Arballo \& Cravino 1999; de la Peña 2010), incubación (Taylor 1998), pichones (Housse 1945) y desarrollo gonadal (Ruiz 1993). Aquí documentamos observaciones sistemáticas de varios aspectos de la biología reproductiva de ambas especies de taguas en un área de uso agrícola del centro-sur de Chile y enfatizamos su importancia para la conservación de los ecosistemas dulceacuícolas.

\section{MATERIALES Y MÉTODOS}

Durante dos temporadas, desde septiembre a diciembre 2008 y 2009, estudiamos la reproducción de una población de taguas residentes en la laguna Santa Elena (36 $48^{\circ} 14^{\prime}$ 'S, $72^{\circ} 23^{\prime} 04^{\prime \prime}$ ), provincia de Nuble, centro-sur de Chile. La laguna Santa Elena (Fig. 1) es un cuerpo natural de agua relativamente pequeño (160 ha) que se encuentra en un área de uso agrícola y forestal donde existen plantaciones de remolacha (Beta vulgaris Linnaeus 1758), trigo (Tritricum spp.), frambuesa (Rubus spp.), arándano (Vaccinium spp.), pino (Pinus radiata D. Don 1825) y eucalipto (Eucalyptus spp.). La vegetación terrestre que bordea la laguna está constituida por especies nativas tales como espino (Acacia caven Molina 1810), arrayán (Luma apiculata Burret 1941), boldo (Peumus boldus Molina 1782), litre (Lithraea caustica Hook. \& Arn. 1833), maqui (Aristotelia chilensis Stuntz 1914) y patagua (Crinodendron patagua Molina 1782), y por especies introducidas tales como pino, álamo (Populus simonii Carrière 1867), sauce amargo (Salix humboldtiana Willdenow 1806), sauce llorón (S. babylonica Linnaeus 1753), sauce mimbre (S. viminalis Turczaninow 1854), aromo australiano (Acacia melanoxylon $\mathrm{R}$. Br. 1813), aromo francés (A. dealbata Cunningham1825) y retamo (Teline monspessulana K. Koch 1869). La vegetación acuática que cubre los bordes está compuesta principalmente por juncos (Juncus procerus E. Mey 1828) (González-Acuña et al. 2004).

La búsqueda de los nidos fue realizada en dos zonas de la laguna (Fig. 1), las cuales fueron escogidas debido a la buena accesibilidad para el investigador y la existencia de juncales. Cada sitio nido encontrado fue marcado para su posterior reconocimiento utilizando una cinta de color atada a la parte superior de los juncos. Después de su hallazgo, los sitios nidos fueron visitados cada tres días para reducir perturbaciones en el nido. En ambos periodos reproductivos registramos la siguiente información: (1) características de los nidos, (2) tamaño de nidada, (3) características de los huevos, (4) duración de la incubación, (5) variación del peso de los huevos, (6) éxito de eclosión, y (7) características de los pichones. El éxito de eclosión se estimó con corrección de Mayfield (1975) [1-(número de huevos perdidos / número de días de exposición)], dando como resultado la tasa de sobrevivencia diaria de los huevos; para obtener una estimación de la supervivencia de los huevos durante el período de anidación, la tasa de supervivencia diaria se elevó a la potencia equivalente a la cantidad promedio de días (d) de incubación. Este método evita el sesgo positivo inherente al éxito aparente del nido, mediante la estimación de tasas de supervivencia diaria usando el número de días de exposición, eliminando la necesidad de monitorear los nidos desde el inicio de la postura. También se calculó el éxito de eclosión utilizando el método tradicional (número de huevos eclosionados / número de huevos puestos), para posibilitar la comparación con otros estudios en los que se emplea este método. Los nidos fueron caracterizados por variables morfométricas, estructurales y espaciales. Las variables morfométricas y estructurales incluyeron: (i) material de construcción, (ii) forma de la plataforma y (iii) alto y ancho de la plataforma y de la taza (Fig. 2a). Las variables espaciales incluyeron: (i) altura desde la base superior del nido a la superficie del agua (Fig. 2b) y (ii) sustrato de anidamiento. El tamaño de nidada se definió 
como el número de huevos puestos por nido. Todos los huevos recién puestos fueron caracterizados por su patrón de color, forma, peso y volumen. El volumen de cada huevo fue calculado siguiendo la formula de Hoyt (1979): V = $0,000507 \mathrm{x}$ largo $\mathrm{x} \mathrm{ancho}^{2}$. Debido a que los pichones de taguas son nidífugos fuimos incapaces de estimar el éxito de abandono de las crías vivas desde el nido. Los pichones recién eclosionados fueron descritos siguiendo el patrón de coloración del plumaje, pico y patas. Los resultados fueron analizados utilizando estadística descriptiva (media, desviación estándar [DE] y rango). Para poder comparar las tasas de eclosión entre diferentes años y especies, se calculó la varianza del estimador de Mayfield según (Jonhson 1979) y luego el valor de Z. Además, se aplicó la prueba $t$ de Student para comparar los datos de morfometría de nidos, volumen y peso de los huevos entre $F$. armillata y $F$. rufifrons y la prueba no paramétrica $U$ de Mann-Whitney para comparar el tamaño de la nidada entre las dos especies.

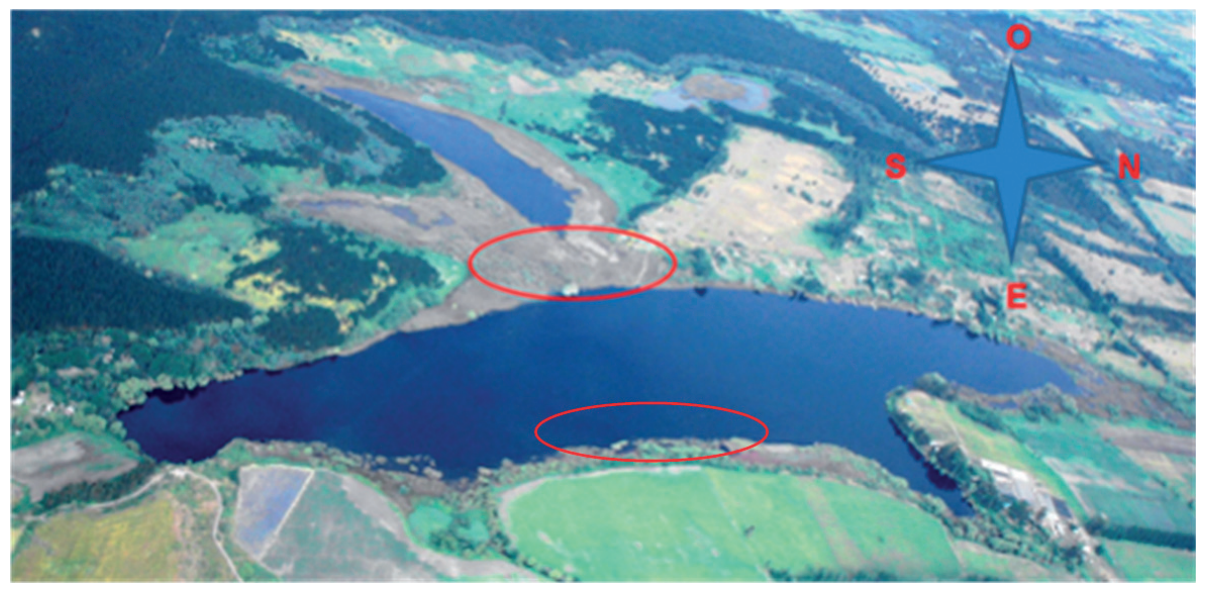

Figura 1. Vista aérea de la laguna Santa Elena indicando los dos sitios donde se realizó el estudio. Foto: Fernando González S.

Figure 1. Aerial view of Santa Elena lagoon indicating the two sites where the study was conducted. Photo: Fernando González S.

\section{RESULTADOS}

Un total de 14 nidos de $F$. armillata y 8 nidos de $F$. rufifrons, fueron monitoreados. Los nidos de F. armillata fueron detectados durante ambos periodos reproductivos ( $\mathrm{n}=$ 7 , respectivamente), mientras que los de F. rufifrons sólo durante el primer periodo. El inicio del periodo reproductivo para ambas especies fue a mediados de septiembre, donde fueron observadas las primeras parejas, extendiéndose hasta fines de enero. La confección de los nidos y postura de huevos fue asincrónica entre parejas de cada especie.

CARACTERÍSTICAS DE LOS NidOS.- La construcción de los nidos en ambas especies de taguas se inició a fines de septiembre. Sin embargo, no se logró determinar si ambos sexos participaron en la construcción de los nidos debido a que tanto F. armillata como F. rufifrons no presentan dimorfismo sexual. No se registró segregación interespecífica de los nidos; ambas especies construyeron sus nidos en la orilla de la laguna en sectores donde hubo gran cantidad de juncos. Los nidos consistieron de plataformas circulares de tamaño similar construidas con juncos secos y en algunos casos rellenas con algas (Tabla 1). Aunque los juncos no fueron fuertemente entrelazados, estos fueron dispuestos de manera entrecruzada siguiendo un patrón circular (Fig. 2a). La taza de cada nido fue también claramente circular y poco profunda (Tabla 1). En el caso F. armillata la taza representó entre $50-70 \%$ de la superficie de la plataforma, y en $F$. rufifrons ca. 55\% (Tabla 1). El fondo de cada taza estuvo bien compactado, relleno con algunas plumas y sin excrementos. Aunque el tamaño medio de los nidos de $F$. armillata varió entre los dos periodos reproductivos estudiados, no se encontró una diferencia estadísticamente significativa (largo: $t=0,17$; ancho: $t=0,21 ; \mathrm{P}>0,05 ; \mathrm{n}=14$ ). Tampoco se registró reutilización de nidos durante el segundo periodo reproductivo. Ambas especies establecieron sus plataformas de nidificación a una altura similar con respecto al espejo de agua. La altura media de los nidos de $F$. armillata con respecto al espejo de agua fue de 16,6 $\pm 2,4 \mathrm{~cm}$ (media \pm $\mathrm{DE}$; rango $=14,0-20,5 \mathrm{~cm})$ y $16,5 \pm 4,3 \mathrm{~cm}(\mathrm{rango}=11,0-$ $24,2 \mathrm{~cm}$ ) durante el primer y segundo año, respectivamente. En el caso de $F$. rufifrons, en la primera temporada fue 17,7 $\pm 7,1 \mathrm{~cm}($ rango $=12,0-31,0 \mathrm{~cm})$. Algunos de los nidos de $F$. armillata y $F$. rufifrons presentaron rampas de acceso (n $=4 \mathrm{y} \mathrm{n}=1$, respectivamente). No se observaron diferencias significativas entre ninguna de las variables morfológicas 
registradas para los nidos de ambas especies (largo: $t=0,68$; ancho: $t=0,87$; largo taza: $t=0,35$; ancho taza: $t=0,65$; profundidad de taza: $t=0,10$; distancia base nido agua: $t=$ $0,72 ; \mathrm{P}>0,05)$.

Como dato curioso, durante el segundo periodo reproductivo de $F$. armillata, un nido fue construido sobre un sauce (Salix sp.) que emergía de la laguna. El nido, fabricado con una mezcla de juncos verdes y secos, se encontraba sobre el nivel del espejo de agua (altura $=24$ $\mathrm{cm})$ y visiblemente expuesto.

TAMAÑO DE NIDADA.- El tamaño de nidada de F. rufifrons fue mayor que el de $F$. armillata $(\mathrm{U}=51,5 ; \mathrm{P}<0,05)$. F. rufifrons tuvo $6,3 \pm 1,5$ huevos por nido (media $\pm \mathrm{DE}$; rango $=4-8$; $\mathrm{n}=8$ ), mientras que $F$. armillata tuvo $3,7 \pm 0,9$ (rango $=$ $3-5 ; \mathrm{n}=7$ ) y $3,6 \pm 0,5$ (rango $=3-4 ; \mathrm{n}=7$ ) huevos por nido, durante el primer y segundo periodo reproductivo, respectivamente.
CARActerísticas de los huevos.- Tanto los huevos de $F$. armillata como de $F$. rufifrons presentaron una forma ovalada (Figs. 2c y 2d). Los huevos de $F$. armillata tendieron a ser más grandes (Tabla $2 ; t=2,76, \mathrm{P}<0,05 ; \mathrm{n}=24$ ) y más pesados que los de $F$. rufifrons $(t=2,74 ; \mathrm{P}<0,05)$. Un aspecto que llamó la atención en nuestro estudio fue la variación interanual en el tamaño de los huevos de $F$. armillata, siendo los huevos de varias parejas más pequeños durante el segundo periodo reproductivo (Tabla 2; $t=4,10 ; \mathrm{P}<0,05$ ). En cuanto a la coloración, los huevos de $F$. armillata y $F$. rufifrons tuvieron patrones distintivos. La superficie externa de los huevos de F. armillata tuvo color blanco crema con pintas y manchas dispersas que variaron desde violeta pálido a rojo marrón oscuro (Fig. 2c). En cambio, los huevos de $F$. rufifrons presentaron una superficie externa de color beige oscuro con un fino puntillado marrón oscuro distribuido sobre toda la superficie, observándose una concentración de pintas, líneas y manchas de color violeta pálido a rojo marrón en el polo más amplio (Fig. 2d).
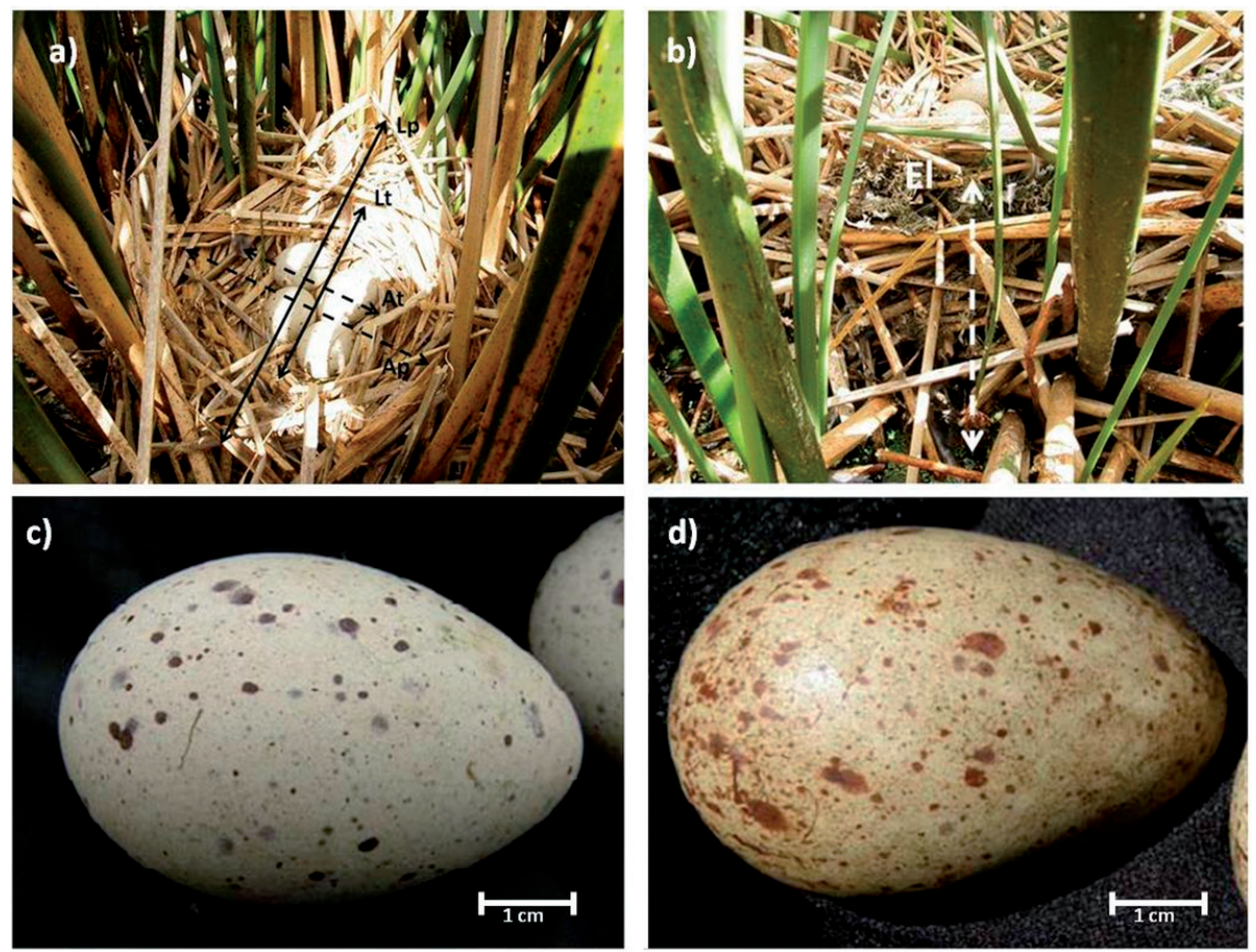

Figura 2. Variables morfométricas y espaciales de los nidos y huevos de F. armillata (en la foto) y F. rufifrons: a) Lp = largo de la plataforma, $\mathrm{Ap}=$ ancho de la plataforma, $\mathrm{Lt}=$ largo de la taza, At = ancho de la taza; b) El = elevación de la plataforma desde la superficie del agua; c) huevo de F. armillata y d) huevo de F. rufifrons colectados en la laguna Santa Elena, provincia de Nuble, centro-sur de Chile (fotos: Ma. Carolina Silva de la Fuente).

Figure 2. Morphometric and spatial variables of the nests and eggs of F. armillata (pictured) and F. rufifrons: a) Lp = length of the platform, $\mathrm{Ap}=$ width of the platform, $\mathrm{Lt}=$ length of the cup, At = width of the cup; b) El = elevation of the platform from the water's surface, c) egg F. armillata and d) egg F. rufifrons collected in the lagoon of Santa Elena, Nuble province, south-central Chile (Photo: María Carolina Silva de la Fuente). 
Tabla 1. Características de los nidos de F. armillata y F. rufifrons en la laguna Santa Elena, centro-sur de Chile. $\mathrm{N}=$ número de nidos.

TABLE 1. Nest characteristics of F. armillata and F. rufifrons on the Santa Elena lagoon, south-central, Chile. $\mathrm{N}=$ nest numbers.

\begin{tabular}{|c|c|c|c|c|c|c|}
\hline \multirow[b]{3}{*}{ Medidas (cm) } & \multicolumn{4}{|c|}{ F. armillata } & \multirow{2}{*}{\multicolumn{2}{|c|}{$\begin{array}{c}\text { F. rufifrons } \\
2008(\mathrm{~N}=8)\end{array}$}} \\
\hline & \multicolumn{2}{|c|}{$2008(\mathrm{~N}=7)$} & \multicolumn{2}{|c|}{$2009(\mathrm{~N}=7)$} & & \\
\hline & Media \pm DE & Rango & Media $\pm \mathrm{DE}$ & Rango & Media $\pm \mathrm{DE}$ & Rango \\
\hline Largo plataforma & $28,4 \pm 5,5$ & $24,0-40,0$ & $31,0 \pm 4,1$ & $23,2-35,5$ & $29,9 \pm 4,1$ & $24,0-35,5$ \\
\hline Ancho plataforma & $29,4 \pm 4,9$ & $23,0-38,0$ & $26,5 \pm 3,4$ & $21,0-31,3$ & $29,5 \pm 4,1$ & $22,0-34,0$ \\
\hline Largo taza & $16,1 \pm 1,0$ & $14,0-17,0$ & $20,1 \pm 1,9$ & $18,0-23,0$ & $17,2 \pm 2,8$ & $13,0-20,0$ \\
\hline Ancho taza & $16,0 \pm 0,8$ & $15,0-17,5$ & $19,0 \pm 3,5$ & $13,0-23,5$ & $15,6 \pm 1,9$ & $13,0-17,5$ \\
\hline Profundidad taza & $4,8 \pm 1,5$ & $2,7-7,4$ & $6,3 \pm 2,3$ & $3,0-9,2$ & $6,3 \pm 1,6$ & $4,0-9,0$ \\
\hline
\end{tabular}

TABLa 2. Características de los huevos de F. armillata y F. rufifrons en la laguna Santa Elena, centro-sur de Chile. $\mathrm{N}=$ número de huevos.

TABLE 2. Eggs characteristics of F. armillata and F. rufifrons on the Santa Elena lagoon, south-central, Chile. $\mathrm{N}=$ eggs numbers

\begin{tabular}{|c|c|c|c|c|c|c|}
\hline \multirow[b]{3}{*}{ Medidas } & \multicolumn{4}{|c|}{ F. armillata } & \multirow{2}{*}{\multicolumn{2}{|c|}{$\begin{array}{c}\text { F. rufifrons } \\
2008(\mathrm{~N}=28)\end{array}$}} \\
\hline & \multicolumn{2}{|c|}{$2008(\mathrm{~N}=23)$} & \multicolumn{2}{|c|}{$2009(\mathrm{~N}=23)$} & & \\
\hline & Media $\pm \mathrm{DE}$ & Rango & Media $\pm \mathrm{DE}$ & Rango & Media $\pm \mathrm{DE}$ & Rango \\
\hline Largo (mm) & $56,2 \pm 3,0$ & $50,3-61,7$ & $48,8 \pm 10,1$ & $34,9-59,0$ & $54,4 \pm 2,4$ & $42,6-60,0$ \\
\hline Ancho (mm) & $38,3 \pm 1,6$ & $36,1-41,7$ & $30,9 \pm 10,0$ & $16,9-41,6$ & $37,1 \pm 1,2$ & $33,1-39,0$ \\
\hline Volumen $\left(\mathrm{cm}^{3}\right)$ & $1,1 \pm 0,1$ & $0,9-1,3$ & $0,8 \pm 0,4$ & $0,3-1,2$ & $1,0 \pm 0,1$ & $0,8-1,1$ \\
\hline Peso (g) & $47,5 \pm 5,6$ & $40,9-57,7$ & $44,5 \pm 1,9 *$ & $42,2-48,5$ & $47,5 \pm 5,3$ & $36,5-58,1$ \\
\hline
\end{tabular}

$* \mathrm{~N}=10$

Postura de huevos E INCUBACIÓN.- El inicio de la postura de huevos para ambas especies ocurrió a fines de septiembre, registrándose las últimas posturas a fines de diciembre. Debido a que las taguas no fueron marcadas no se pudo determinar si tuvieron más de una nidada por temporada. El tiempo de incubación de F. armillata y F. rufifrons sólo pudo ser estimado sobre la base de un nido para cada especie. Debido a que casi todas las parejas ya habían comenzado la incubación cuando iniciamos las observaciones, fue difícil registrar nidos en estado pre-incubación. Ambas especies extendieron su periodo de incubación por un tiempo similar, siendo 27 días en $F$. armillata y 26 días en $F$. rufifrons. Durante la incubación, las taguas se mostraron agresivas ante la presencia de otras taguas y otras especies de aves que se acercaron a los nidos.

VARIACIÓN EN EL PESO DE LOS HUEVOS.- A lo largo del periodo de incubación detectamos una disminución del peso de los huevos de ambas especies de taguas. Los huevos de $F$. armillata $(\mathrm{n}=23)$ tuvieron una pérdida diaria promedio de
$0,2 \pm 0,1 \mathrm{~g}$ alcanzando un pérdida de $6,2 \pm 2,4 \mathrm{~g}$ al final de la incubación $(13,3 \%$ del peso promedio inicial; rango $=$ $10,9-15 \%)$. Los huevos de F. rufifrons $(\mathrm{n}=27)$ perdieron diariamente $0,14 \pm 0,03 \mathrm{~g}$ alcanzando una pérdida total de $3,8 \pm 0,8 \mathrm{~g}$ al término de la incubación $(8,9 \%$ del peso promedio inicial; rango $=8,1-10,4 \%$ ).

Éxito DE ECLOSIÓN.- El éxito de eclosión de $F$. armillata fue menor en el primer periodo reproductivo (método de Mayfield: 46\%; método tradicional: $88,5 \%$ ) comparado con el segundo (método de Mayfield: 62\%; método tradicional: $96 \%)$, pero no hubo diferencias significativas $(Z=0,001 ; P$ $>0,05)$. Aunque no hubo abandono de nidos, tres huevos desaparecieron por causas desconocidas durante el primer periodo y un huevo no eclosionó durante el segundo periodo. F. rufifrons presentó un éxito de eclosión significativamente mayor (método de Mayfield: 85\%; método tradicional: 98\%) que $F$. armillata (método de Mayfield: $46 \%$; método tradicional: $88,5 \%$ ) para el mismo periodo reproductivo $(\mathrm{Z}$ $=2,73 ; \mathrm{P}<0,05)$. Tampoco se registró abandono de nido 
en esta especie y sólo un huevo no eclosionó. Al abrir los huevos no eclosionados se comprobó que eran infértiles.

CARACTERÍsticas de los Pichones.- Debido a que los pichones de taguas son nidífugos sólo fue posible medir y caracterizar a tres ejemplares de cada especie durante el día que ocurrió la eclosión. Aunque los pichones de $F$. armillata fueron algo más pesados (media $\pm \mathrm{DE}=32,7 \pm 6,5 \mathrm{~g}$ ) que los de F. rufifrons (media $\pm \mathrm{DE}=28,7 \pm 0,4 \mathrm{~g}$ ), los pichones de $F$. rufifrons tendieron a ser de tamaño mayor (media $\pm \mathrm{DE}$ largo total $=100,8 \pm 2,2 \mathrm{~mm}$ ) comparados con $F$. armillata (media $\pm \mathrm{DE}$ largo total $=115,9 \pm 40,2 \mathrm{~mm}$ ). Esta inconsistencia entre tamaño y peso posiblemente fue influenciada por el número reducido de la muestra. Por otra parte, los pichones de ambas especies presentaron diferencias evidentes en los patrones de coloración. Aún cuando en ambos casos los pichones nacieron con plumones negros en la mayor parte del cuerpo, ellos se diferenciaron por el color del pico, negro en $F$. armillata y rojo pálido en $F$. rufifrons; el escudo frontal rojo pálido en $F$. rufifrons; piel de la cabeza con zona supraocular azul piedra pálido y corona roja en $F$. armillata y zona supraocular verde agua y corona anaranjada en $F$. rufifrons; patas negras en $F$. armillata y amarillas en F. rufifrons.

\section{DISCUSIÓN}

El número de nidos encontrados para cada especie en la laguna Santa Elena se condice con sus abundancias respectivas en el sitio de estudio, durante el periodo de muestreo. Aunque en el periodo 2002 y 2003 F. armillata fue más abundante que $F$. rufifrons (20,3 taguas/hora vs 2,5 taguas/hora; González-Acuña et al. 2004), esta figura cambió durante el periodo 2008 -2009 cuando ambas especies presentaron una abundancia similar ( $F$. armillata $=14,2$ taguas/hora; F. rufifrons $=12,3$ taguas/hora). A pesar que el número de F. rufifrons se mantuvo sin grandes variaciones durante la segunda temporada reproductiva, no se encontraron sus nidos ni aves juveniles en ninguno de los dos sitios monitoreados en la laguna. Esto podría explicarse por la disminución en la cantidad y densidad de juncos durante la segunda temporada de monitoreo, cuando además se registró un aumento de algas flotantes. De acuerdo a Arballo \& Cravino (1999), ésta especie prefiere construir su nido entre la vegetación acuática, generalmente bien oculta, utilizando aquellas zonas más densas adyacentes a charcas pequeñas.

CARActerísticas de los Nidos.- Las características de los nidos de ambas especies de taguas en nuestro sitio de estudio fueron muy similares con aquellas documentadas para otras localidades; varios autores han descrito los nidos como tazas redondas constituidas por juncos entrecruzados transversalmente, bien apretados y asentados entre el juncal vivo (Housse 1945; Goodall et al. 1951; Taylor 1998;
Arballo \& Cravino 1999; de la Peña 2010). Coincidiendo con nuestras observaciones, Housse (1945) también detectó nidos con rampas de acceso utilizadas por los pichones para salir y entrar del nido. Debido a que los nidos de ambas especies de taguas son muy similares es difícil diferenciarlos en ausencia de la especie respectiva (Goodall et al. 1951). De hecho, en la laguna Santa Elena los nidos de F. armillata y F. rufifrons presentaron una gran similitud morfométrica y estructural. Al igual que en nuestro caso, Housse (1945) también describe un nido de $F$. armillata localizado sobre el nivel del espejo de agua, aunque a una altura algo mayor (34 cm). Tanto el hallazgo de Housse (1945) como el nuestro sugieren que $F$. armillata tiene la capacidad de construir sus nidos a un rango variable de alturas. Aunque los nidos construidos a alturas muy elevadas pueden exponer los huevos, pichones y adultos ante depredadores aéreos, estos parecen ser raros y posiblemente reflejan la escasez de sitios vacantes para nidificación o la inexperiencia de parejas jóvenes.

TAMAÑo DE NIDADA.- Los tamaños de nidada de $F$. armillata documentados aquí tendieron a ser más pequeños que aquellos descritos para otras localidades de Chile y Sudamérica. En Chile, varias descripciones previas indican nidadas de 6-7, 4-7 ó 4-8 huevos (Housse 1945; Goodall et al. 1951; Rottmann 1995). Arballo \& Carvino (1999) y de la Peña (2010) describen tamaños de nidada de $6-8$ y 2 -7 huevos para Uruguay y Argentina, respectivamente. Por otra parte, el tamaño de nidada de $F$. rufifrons casi duplicó la de $F$. armillata difiriendo con lo documentado por otros autores (Goodall et al. 1951; Rottmann 1995; de la Peña 2010), quienes afirman que ambas especies tiene tamaños de nidada similar. Posiblemente, las diferencias encontradas con respecto a otras localidades estén influenciadas por variaciones geográficas, factores climáticos, productividad de cada sitio o condición individual y edad de las aves (e.g., Steubing et al. 1980; Welty \& Baptista 1988). Estudios adicionales que incluyan un mayor número de nidos y sitios reproductivos serán necesarios para demostrar si las diferencias entre especies son consistentes.

Características de los huevos.- Las morfometría de los huevos de ambas especies en la laguna Santa Elena coinciden con aquella documentada previamente para Chile central por Housse (1945) y Goodall et al. (1951). En otras localidades de Sudamérica los huevos de ambas especies también presentaron tamaños muy similares (F. armillata: largo $\mathrm{x}$ ancho $=58-58,8 \times 37,1-39,4$ $\mathrm{mm} ; F$. rufifrons: largo $\mathrm{x}$ ancho $=51,5-55,5 \times 37,1$ - 37,2 mm; Taylor 1998; Arballo \& Cravino 1999). Sin embargo, debido a que estas medidas pueden variar entre temporadas, como sucedió con $F$. armillata, el tamaño de éstos no parece ser un buen indicador para diferenciar los nidos de ambas especies. El peso medio inicial de los 
huevos de F. armillata y F. rufifrons fue cercano a los pesos documentados por de la Peña (2010) quien documenta valores similares para ambas especies $(F$. armillata: media $=48,7 \mathrm{~g}[\mathrm{n}=4] ;$ F. rufifrons: $48,6 \mathrm{~g}[\mathrm{n}=3])$. Al comparar ambos periodos reproductivos de $F$. armillata, notamos una diferencia importante en el tamaño y peso de sus huevos los cuales fueron más pequeños y más livianos en la segunda temporada. Desconocemos las causas reales de este cambio, pero algunos factores potenciales que podrían haber influido son la edad y/o estado nutricional de las aves. Welty \& Baptista (1988) señalan que aves jóvenes comúnmente ponen huevos más pequeños que las aves adultas. Sin embargo, debido a que no se pudo determinar la edad de las parejas durante ambos periodos reproductivos no podemos sostener esta presunción. El estado nutricional vinculado a la productividad del sitio pudo haber sido otro factor que condujo a la disminución del tamaño de los huevos. Aunque no tuvimos información sobre el nivel de productividad de la laguna Santa Elena durante ambos periodos reproductivos, el hecho que durante el segundo año $F$. rufifrons no haya nidificado allí sugiere que pudo haber ocurrido una disminución de la calidad o cantidad de algún tipo de recurso necesario para la nidificación. De acuerdo a nuestra información, el tamaño de nidada no parece haber sido un factor causante de la variación de tamaño de los huevos ya que durante ambos periodos reproductivos $F$. armillata tuvo tamaños de nidada similares.

Los huevos de ambas especies presentaron patrones de coloración notablemente distintas. Tales características son consistentes con descripciones anteriores hechas por otros autores. Housse (1945) afirma que los huevos de F. armillata tienen la superficie externa de color blanco crema grisácea salpicadas de pintas finas negras y violáceas y pecas negromoradas o violeta pálido. Goodall et al. (1951) señalan que el fondo de la superficie externa es café claro con gran número de pintas café oscuro, negras o rojizas y con ausencia de manchas gruesas. Housse (1945) menciona que la superficie externa de los huevos de $F$. rufifrons es color blanco amarillento salpicada de pintas finas de color violeta y máculas dispersas de distinto tamaño de color morado o café oscuro. Aunque Goodall et al. (1951) afirman que los huevos de ambas especies son distinguibles por su color, ellos hacen una descripción pobre de los huevos de F. rufifrons señalando solamente que tienden a ser de color piedra. En general, nuestras descripciones también coinciden con aquellas dadas por de la Peña (2010) para Argentina y por Arballo \& Cravino (1999) para Uruguay. Curiosamente, ninguno de los autores anteriores destacó la concentración de manchas en el polo más amplio de los huevos de F. rufifrons. Dadas las diferencias evidentes, los patrones del color externo de los huevos pueden ser utilizados confiablemente para distinguir los nidos de ambas especies de taguas.
Período de Postura e InCUBACIÓN.- Nuestras observaciones acerca de la fenología reproductiva de $F$. armillata y $F$. rufifrons coinciden con lo mencionado por Goodall et al. (1951). Estos autores afirman que el periodo de postura para ambas especies comienza en octubre y termina usualmente en noviembre, pero a menudo se prolonga hasta diciembre o enero. En nuestro estudio no logramos identificar si ambos sexos participan en la incubación. Aunque no existe información para las especies estudiadas, en otras especies de Fulica, ambos miembros de la pareja participan en la incubación. Sin embargo, el macho invierte menos tiempo en el cuidado parental que la hembra, observándose en el nido con mayor frecuencia en la noche (Fredrickson 1970). La duración de la incubación de $F$. armillata fue similar a la documentada por Taylor (1998), quién menciona una duración de $24-25$ días. En el caso de F. rufifrons, la extensión de su periodo de incubación en nuestro sitio de estudio coincidió con lo documentado por Escalante (1991) para Montevideo, Uruguay (25 días).

VARIACIÓN EN EL PESO DE LOS HUEVOS.- La disminución en el peso de los huevos durante el periodo de incubación en $F$. armillata y $F$. rufifrons también ha sido detectada en otras especies de aves (e.g., Zicus et al. 2004). Varios autores han observado que tal disminución está determinada esencialmente por la pérdida de agua causada por factores intrínsecos y extrínsecos a lo largo de la incubación (e.g., Ar \& Rahn 1980; Booth \& Seymour 1987; Kern et al. 1992). Sotherland et al. (1980) afirman que la absorción de calcio por el embrión produce adelgazamiento de la cáscara facilitando la pérdida de humedad dentro del huevo. En algunas especies, el aumento de la temperatura del huevo con el tiempo de incubación provoca una presión creciente del vapor de agua y consecuentemente pérdida de peso (Caldwell \& Cornwell 1975). Además, el aumento de temperatura dentro del nido como consecuencia de modificaciones ambientales puede inducir un aumento en la porosidad de la cáscara incrementando a la vez la conductancia del vapor de agua (Carey 1980; Sotherland et al. 1980; Arad et al. 1988). El porcentaje de pérdida de peso de los huevos de $F$. armillata se ajustó al valor típico de 15\% (Ar \& Rahn 1980). Sin embargo, en el caso de $F$. rufifrons el porcentaje de peso perdido cayó bajo este valor, lo que podría explicarse por las diferencias de tamaño de los huevos de ambas especies. Ar et al. (1974) detectaron que entre especies la conductancia del vapor de agua aumenta con el tamaño del huevo. Así, sería esperable que los huevos de $F$. armillata pierdan proporcionalmente más peso que los huevos de F. rufifrons.

Éxito DE EClosión.- Hasta donde sabemos, el éxito de eclosión de los huevos de F. armillata y $F$. rufifrons no ha sido documentado anteriormente en la literatura. Como ocurre en estas especies, otras especies de taguas también 
exhiben un éxito de eclosión alto (77\%, Rizzi et al. 1999; 81,8\%, Uzun et al. 2010). Sin embargo, estos estudios sólo utilizaron el método tradicional de cálculo por lo cual tales éxitos de eclosión podrían estar sobreestimados. El comportamiento agresivo, típico de las taguas (Navas 1960; Couve \& Vidal 2003; Martínez \& González 2004), podría ser una de las razones del alto éxito de eclosión en $F$. armillata y $F$. rufifrons. Cabe destacar que la agresividad de las taguas correspondió claramente a una interacción interespecífica, no registrándose agresiones hacia nosotros cuando nos aproximamos a los nidos. Por otra parte, la cantidad de huevos infértiles en los nidos de $F$. armillata y $F$. rufifrons fue baja. Al igual que en nuestro caso, Rizzi et al. (1999) y Uzun et al. (2010) también encontraron una proporción baja de huevos infértiles en los nidos de $F$. atra $(0,5-7,6 \%)$. Los huevos no eclosionados fueron encontrados flotando cerca del nido después de que el resto de los huevos eclosionó. Usualmente, los huevos no eclosionados y restos de huevos eclosionados son removidos del nido por las aves adultas presumiblemente para evitar la presencia de depredadores atraídos por el olor causado por la descomposición orgánica (Wallace 1955).

CARacterísticas de los Pichones.- En términos generales, nuestra descripción de los pichones de $F$. armillata y $F$. rufifrons es consistente con aquellas hechas por otros autores (Housse 1945; de la Peña 2010). Particularmente para Chile, Housse (1945) menciona que los pichones de F. armillata nacen con plumones negros en el cuerpo, plumón rojizo en la garganta y cuello, mancha roja en el pico, escudo frontal rojo castaño, y color azul alrededor de los ojos. De acuerdo a este mismo autor, los pichones de $F$. rufifrons nacen con plumones negros, pico amarillento, escudo frontal rojizo y plumas rojas en el pecho y cuello. Nuestra descripción, algo más detallada de los pichones, puede ser una guía útil para reconocer los sitios de nidificación de ambas especies de taguas. No obstante, su utilidad puede ser relativa debido a la conducta nidífuga de los pichones.

MANEJO Y CONSERVACIÓN.- Actualmente, muchos sistemas dulceacuícolas de Chile se encuentran sometidos al efecto de las actividades humanas intensivas tales como explotación forestal, acumulación de sustancias agroquímicas o de residuos industriales. El efecto de estas actividades, dado el carácter dinámico de los ambientes lóticos, pueden afectar cualquier parte del ecosistema. La aves, especialmente las acuáticas, juegan un rol importante en el monitoreo de estos ambientes, aportando información valiosa como bioindicadoras del estrés antrópico (Ormerod 1985; Biondi 1993). Un reflejo de éste estrés podría ser la drástica disminución de juncos en la laguna Santa Elena lo que, a la vez, pudo haber conducido a la disminución de la tasa de nidificación de F. rufifrons. Cuevas (1994) evaluó el efecto de la contaminación en la reproducción de $F$. atra detectando que el éxito reproductivo fue mayor en aquellas zonas menos contaminadas. Lo anterior confirma que las aves acuáticas son útiles para dar un panorama general de los ecosistemas que habitan. Por otra parte, la extracción ilegal de huevos, especialmente de especies amenazadas, hecha por lugareños o visitantes aumentaría la amenaza o vulnerabilidad de las aves acuáticas. En la laguna Santa Elena hemos evidenciado la extracción de huevos de cisne de cuello negro (Cygnus melancoryphus Molina 1782) y sospechamos que esto pudo haber sido también la causa de desaparición de los huevos de taguas. El conocimiento sobre los aspectos reproductivos de las especies silvestres es relevante en la planificación seria del manejo y conservación de los ecosistemas naturales. Creemos que parte de la información proporcionada aquí puede ser potencialmente útil para reconstruir la condición original de sistemas que han sido alterados por causas naturales o humanas (e.g., características de los nidos) en programas futuros de restauración ecológica

\section{BIBLIOGRAFÍA}

Ar, A., Paganelli, C.V., Reeves, R.B., Greene, D.G. \& Rahn, H. 1974. The avian egg: water vapor conductance, shell thickness, and functional pore area. Condor 76:153-158.

AR, A. \& RAHN, H. 1980. Water in the avian egg: overall budget of incubation. American zoologist 20:373-384.

Arad, Z., Gavrieli-Levin, I. \& Marder, J. 1988. Adaptation of the pigeon egg to incubation in dry hot environments. Physiological Zoology 61:293-300.

Arballo, E. \& Cravino, J. 1999. Aves del Uruguay: manual ornitológico. Vol. 1. Hemisferio Sur. Montevideo, Uruguay. $466 \mathrm{pp}$.

Biondi, M.; Guerrieri, G. \& Pietrelli, L. 1993. Stress antropogenicied evolzione di una comunità ornitica in una zona umida artificiale dell'Italia centrale. Avocetta 17:2327.

Boотн, D.T. \& Seymour, R.S. 1987. Effect of eggshell thinning on water vapor conductance of malleefowl eggs. Condor 89:453-459.

Caldwell, P.J. \& CoRnwell, G.W. 1975. Incubation behavior and temperatures of the mallard duck. Auk 92: 706-731.

Celis, J. 2006. Humedales y Biodiversidad. Revista Ciencia Ahora 18(9).

CAREY, C. 1980. Adaptation of the avian egg to high altitude. American Zoologist 20: 449-459.

Couve, E. \& Vidal-OjedA, C. 2000. Aves del Canal Beagle: Cabo de Hornos, Islas de los Estados, Islas Diego Ramírez y mares adyacentes. Fantástico Sur Birding. Punta Arenas, Chile. 265 pp.

Cuevas, J.A. 1994. Estudio de una comunidad reproductora de aves acuáticas en un curso fluvial fuertemente antropizado. Actas de las XII Jornadas Ornitológicas Españolas. Pp. 4754.

DE LA PeÑA, M.R. 2010. Nidos de aves argentinas. CD. Universidad Nacional del Litoral, Santa Fe, Argentina.

Escalante, R. 1991. Notas sobre la biología de la reproducción de la gallareta de escudete rojo (Fulica rufifrons, Rallidae). 
Comunicaciones Zoológicas del Museo de Historia Natural de Montevideo 12:1-16.

Figueroa, R.A., Cerda, J. \& Tala, C. 2001. Guía de aves dulceacuícolas de Aysén. Servicio Agrícola y Ganadero de Aysén, Ministerio de Agricultura, Chile. 184 pp.

FJelds̊̊, J. \& Krabbe, N. 1990. Birds of the high Andes: a manual to the birds of the temperate zone of the Andes and Patagonia, South America. University of Copenhagen. Zoological Museum. Copenhagen, Denmark. 876 pp.

FREDRICKSON, L.H .1970. Breeding biology of American Coots in Iowa. Wilson Bulletin 82:445-457.

González-Acuña, D., Benavente, C. \& Figueroa, R.A. 2004. Avifauna de la laguna Santa Elena, región del Bío Bío. Boletín Chileno de Ornitología 10:13-18.

Goodall, J. D., Johnson, A.W. \& Philippi, R. A. 1951. Las aves de Chile: su conocimiento y sus costumbres. Tomo segundo. Platt Establecimientos Gráficos, Buenos Aires, Argentina. $450 \mathrm{pp}$.

Housse, R. 1945. Las aves de Chile en su clasificación moderna: su vida y costumbres. Ediciones de la Universidad de Chile. Santiago, Chile. 384 pp.

Hoyt, D.F. 1979. Practical methods of estimating volume and fresh weights of bird eggs. Auk 96:73-77.

Jaramillo, A., Burke, P. \& Beadle, D. 2003. Birds of Chile. Christopher Helm, London. 240 pp.

Kern, M.D., Cowie, R.J. \& Yeager, M. 1992. Water loss, conductance, and structure of eggs of pied flycatchers during egg laying and incubation. Physiological Zoology 65:1162-1187.

Kushlan, J.A. 1993. Colonial waterbirds as bioindicators of environment change. Colon. Waterbirds 16:223-251.

Martínez, D.E. \& GonzÁLez, G.E. 2004. Aves de Chile: nueva guía de campo. Ediciones del Naturalista. Santiago, Chile. 620 pp.

MAYField, H.F. 1975. Suggestions for calculating nest success. Wilson Bulletin 87:456-466.

Ormerod, S.J., Bollstone, M.A. \& TYler, S.J. 1985. Factors influencing the abundance of breeding Dippers Cinclus cinclus in the catchment of the River Wye, mid-Wales. Ibis 127:332-340.

Rizzi, H., Benyacoub, S., Chabi, Y. \& Banbura, J. 1999. Nesting and reproductive characteristics of coots Fulica atra breeding on two lakes in Algeria. Ardeola 46:179-186.

Rottmann, J. 1995. Guía de identificación de aves de ambientes acuáticos. Unión de Ornitólogos de Chile, Santiago, Chile. $77 \mathrm{pp}$.

RuIZ, J.E. 1993. Estudio ecológico sobre tres especies de taguas residentes en el Santuario de la Naturaleza del Río Cruces. Tesis Licenciatura Medicina Veterinaria, Universidad Austral de Chile. Valdivia, Chile.

Sotherland, P.R., Packard, G.C., Taigen, T.L. \& Boardman, T.J. 1980. An altitudinal cline in conductance of cliff swallow (Petrochelidon pyrrhonota) eggs to water vapor. Auk 97:177-185.

Steubing, L., Ramírez, C. \& Alberdi, M. 1980. Energy content of water-and bog-plant associations in the region of Valdivia. Plant Ecology 43:153-161.

TAYLOR, B. 1998. Rails: a guide to the rails, craks, gallinules and coots of the world. Yale University Press. New Haven, USA. 600 pp.

Uzun, A., Uzun, B. \& Kopis, G. 2010. The effect of clutch size on egg and hatchling mass and measurements in the Common Coot Fulica atra. Ekoloji 19:160-163.

Wallace, G.J. 1955. An introduction to ornithology. MacMillan Company. New York, USA. 512 pp.

Weller, M.W. 1999. Wetland birds: habitat resources and conservation implications. Cambridge University Press, New York, USA. 316 pp.

Welty, J.C. \& Baptista, L. 1988. The life of birds. 4th ed. Saunders College Publishing. Orlando, USA. 720 pp.

Wetmore, A. 1926. Observation of the birds of Argentina, Paraguay, Uruguay and Chile. Bulletin 133. United States National Museum. Washington DC, USA. 448 pp.

Zicus, M.C., Rave, D.P. \& Riggs, M.R. 2004. Factors influencing incubation egg-mass loss for three species of waterfowl. Condor 106:506-516.

Recibido: 12.05 .11

Aceptado: 22.07.11 